\title{
Impact of the epidemic on the world economy
}

\author{
Zihuan Feng ${ }^{1}$, Xun Liang ${ }^{1, *}$, and Binqing $\mathrm{Cai}^{2}$ \\ ${ }^{1}$ School of information, Renmin University of China, China \\ ${ }^{2}$ School of management, Fujian Institute of Technology, China
}

\begin{abstract}
The impact of the epidemic on the global economy has exposed the fragility and complexity of the internal and external economic system, and the global economic situation is not optimistic. Taking the epidemic as an example, this paper analyzed the impact of emergencies on the global economy from multiple perspectives. The outbreak of the epidemic has made most offline businesses shut down overnight. At the same time, it has also led to the rapid development of online economy, such as online education, home office software, video entertainment and so on. The outbreak and spread of the epidemic has promoted the digital upgrading of all walks of life, including online government affairs, commerce, science, education, culture and health organization, operation and maintenance. The era of digital economy has come, and digitization is the general trend.
\end{abstract}

Keywords: Economic situation, Medical care, Digital transformation, Digital economy.

\section{Introduction}

Under the background of novel coronavirus pneumonia, the economy has entered a technical recession and the macroeconomic situation is more complicated. From the historical point of view, from 1637 to date, there have been 10 major economic and financial crises in human history: the tulip crisis in Holland in 1637, the bubble in the South China Sea in 1720, the financial panic in 1837, the financial crisis in the United States, the banking crisis in 1907, the depression in the United States, the global stock market crash, and the Mexico depression. Crisis; the US internet bubble in 2000; the subprime mortgage crisis in 2007 [1]; the impact of COVID-19 in 2020. Economic crisis is a broad concept. For the convenience of research, it can be divided into three concepts: liquidity crisis, financial crisis and economic crisis [2]: liquidity crisis mainly refers to the destruction of cash flow statement and the depletion of cash flow; financial crisis mainly refers to the destruction of balance sheet of financial system; economic crisis includes recession The former is usually accompanied by the decline of macroeconomic indicators such as GDP, employment, investment expenditure, capacity utilization rate, household income, corporate profits and inflation, while the rise of bankruptcy unemployment rate generally refers to two consecutive quarters or more of negative economic growth, while the economic recession lasts longer and the decline of economic activity is greater than any other

* Corresponding author: xliang@ruc.edu.cn 
economic recession If the real GDP drops by more than $10 \%$ and lasts for more than two years, it can be regarded as "economic depression".

Under the background of stock market crash and market liquidity depletion, the central bank can import liquidity to correct the deviation of the market from the macro-economy, protect the cash flow statement, avoid the bankruptcy of banks and financial institutions, block the transmission of liquidity crisis to financial crisis, and protect the balance sheets of enterprises and families. The relationship between opportunity and economic crisis is more complicated.

The latest data from the purchasing managers' index (PMI) shows that exports are still shrinking. Although industrial production, retail sales of consumer goods and fixed asset investment are on the rise, the sustained weakness of domestic demand and the coming global economic recession may restrict economic growth in the second half of 2020 [3].

\section{Global economic situation under the impact of epidemic situation}

Expanding domestic demand can promote the internal circulation through the long-term mechanism of consumption investment, and abandon all kinds of short-term policies to stimulate consumption and stimulate investment, which shows that the strategic position of expanding domestic demand is more basic and important. From the perspective of promoting consumption, the key is to expand the effective demand of residents. The specific long-term measures include three aspects: first, comprehensively grasp the basic strategy of targeted poverty alleviation and targeted poverty alleviation to improve the income of migrant workers and low-income urban bottom residents; second, speed up the reform of basic public services, such as increasing the proportion of education, medical care and pension in the financial expenditure, and strive to promote the development of rural areas. From the perspective of expanding investment, we should promote manufacturing investment, develop industry and build a more productive investment system. In order to adapt to the market-oriented nature of manufacturing investment, we should improve the business environment to improve efficiency, implement tax reduction and fee reduction policies to reduce production and operation costs and promote market development [4].

Since 2020, the global economy has been impacted by the epidemic, which has exposed the fragility and complexity of the internal and external economic system: first, there is panic in the market. The market has fallen into the panic cycle of selling assets for many times, and the safe assets and risky assets generally fell. In addition to the drastic fluctuations in the international crude oil and capital markets, the price of gold has also fallen in recent years. In order to alleviate the liquidity shortage, institutional investors are forced to sell all assets: they are selling risky assets as well as hedging assets. This means that people only focus on liquidity rather than value, which is a common psychological feature in crisis, and directly leads to capital outflow and devaluation of local currency in emerging economies. The Federal Reserve and central banks around the world have reached a consensus on "releasing water" to deal with the liquidity crisis and protect the cash flow statement. Many countries have introduced monetary easing policies and financial rescue plans. Financial institutions are an important link to prevent the development of liquidity crisis into the domino effect of financial crisis. With the improvement of international regulatory standards after the 2008 financial crisis, the capital adequacy ratio of major global banks is relatively high, the asset liability structure is relatively stable, and the global financial system has a certain anti shock ability. In the first quarter, although the market value of 21 major banks in the world dropped by nearly 5.6 trillion yuan, the decline of Barclays Bank and that of Citigroup ranking among the top two market performance declines (see Table 1), the impact on the financial system was 
acceptable. Among them. Third, there are signs of bankruptcy in key enterprises. The increasingly severe border blockade measures among major countries make the operation of global industrial chain suffer "hard decoupling" from both sides of supply and demand, resulting in the "sudden stop" of partial operation of the international division of labor system. The GDP of the economies seriously affected by the epidemic accounts for $80 \%$ of the world's total, and the supply chain affected accounts for $90 \%$ of the world's total. Since the outbreak of the epidemic, more than 1000 enterprises have issued bankruptcy announcements. Most of the bankrupt enterprises are closely related to the hotel industry and tourism industry, and even spread from the industrial chain to the foreign trade industry and transportation industry, followed by the small and medium-sized enterprises that provide supporting services for these industries. In the industrial structure of the United States, the service industry accounts for as much as $80 \%$, which is the area most seriously affected by the epidemic. The employment proportion of service industry is high, the possibility of large-scale unemployment is high, the income is mainly cash, and the cash flow problem affects the debt. The enterprises impacted by the epidemic extend from service industry to manufacturing industry and financial industry. However, innovative and forward-looking enterprises, artificial intelligence, networking, high degree of digitization enterprises, such as live sales, online video and other industries, were relatively less affected by the epidemic, and even rose against the trend. Fourth, the unemployment rate is deteriorating.

Table 1.2020 Q1 capital market performance of global systemically important banks.

\begin{tabular}{|c|c|c|c|}
\hline Bank & Decline (\%) & Bank & Decline (\%) \\
\hline Barkley bank & 51.91 & $\begin{array}{c}\text { Mitsubishi UFJ Financial } \\
\text { Group }\end{array}$ & 32.6 \\
\hline Citibank & 47.03 & HSBC Holdings & 28.14 \\
\hline Wells Fargo & 46.46 & UBS group & 26.39 \\
\hline Santander bank & 43.1 & Mizuho financial group & 25.24 \\
\hline Credit Suisse Group & 39.76 & Royal Bank of Canada & 22.08 \\
\hline Bank of America & 39.6 & Deutsche Bank & 16.84 \\
\hline JPMorgan Chase & 35.06 & $\begin{array}{c}\text { Industrial and Commercial } \\
\text { Bank of China }\end{array}$ & 12.41 \\
\hline State Street bank & 33.31 & $\begin{array}{c}\text { Bank for economic } \\
\text { construction }\end{array}$ & 12.31 \\
\hline Morgan Stanley & 33.28 & Agricultural bank & 8.67 \\
\hline Bank of New York Mellon & 32.88 & Bank of China & 5.69 \\
\hline Goldman Sachs Group & 32.73 & & - \\
\hline
\end{tabular}

(Source: Hecent bank)

\section{The impact of the epidemic on different industries}

A global epidemic of new coronavirus pneumonia has shut down businesses on most offline businesses overnight. At home, online businesses such as online shopping, home fitness, telecommuting, sharing employees, unmanned distribution, cloud classroom, online medical service, and community purchasing have ushered in unexpected outbreaks [5].

Under the epidemic situation, the medical and health sector has the fastest response and the most direct demand outbreak point. During the epidemic period, internet medical related companies, such as Ali health, Ping'an good doctor, micro medicine, Medical Association, clove doctor and other platforms, have opened online free clinic to screen 
common cold and new pneumonia, relieve offline pressure, reduce cross infection, and let more people experience Internet Medical. According to the public health data, as of January 30 , the number of online free clinic users has exceeded 2.8 million, the number of doctors has exceeded 1000 , and the average daily number of online doctors participating in online free clinic has exceeded 100. In this wave of actions, internet medical enterprises have proved their deployment, organization and operation capabilities, and proved their credibility and applicability to the B-end and C-end (see Figure 1). For example, the hospital information enterprise improves the hospital's awareness of telemedicine, which not only provides the enterprise with customer access and education market, but also verifies its value in front of the management and control department and the C-end public. It is a good opportunity to go out of the circle. The epidemic has given telemedicine a catalyst. The attitude of many hospitals to telemedicine has changed from resistance and indifference to trying to accept and benefit from it. "Information enterprises have helped a lot of hospitals to make Internet transformation and increase online consultation, but many hospitals have not used it. This time, we helped the hospital set up channels and services, trained doctors, and relieved the pressure of the hospital's offline outpatient service." In the next 2 to 3 years, telemedicine will be further popularized, especially in places where medical resources are lacking. It is a supplement to hierarchical diagnosis and treatment, and will be conducive to the rational allocation of medical resources [6].

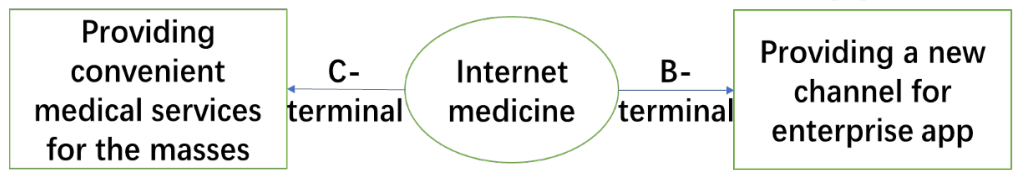

Fig. 1. Online medical B / C terminal.

After the outbreak of the epidemic, offline training institutions stopped business in order to avoid gathering, and primary and secondary schools all over the country postponed their opening. The offline training institutions that have to help themselves begin to turn to online teaching, and the online education enterprises that urgently need to grow are paying close attention to this opportunity. Online education is back in the air again [7]. Classin, Edusoho, Mukun Technology and other online live broadcasting platforms are free to open their technical capabilities. Hundreds of thousands of students have begun to access the online live broadcasting system "cloud classroom" in succession, and tens of thousands of teachers have also transferred from offline to online. Many enterprises also launch free courses, reducing the threshold of experience will inevitably increase the utilization rate of online education products, and bring great opportunities for enterprises to retain and transform.

From the perspective of industry distribution of foreign investment in digital economy, it mainly focuses on online industries, such as software customization, financial technology, big data, e-commerce and network security. In the epidemic situation, the business of enterprises in the online field is relatively less impacted. On the contrary, some industries may benefit from people's many activities turning to the online. Although these industries may be dragged down by the deterioration of macro-economy in the short term, the medium and long-term trend is favorable for the development of these industries.

\section{Trend and characteristics of public opinion under the background of COVID-19}

In the information age, the Internet has become a key place for the dissemination of public opinion. During the epidemic prevention and control period, various kinds of false 
information can be spread in a wider range and at a faster speed through mobile Internet channels, causing huge social impact. Since it is difficult for the public to timely obtain the true data related to the epidemic provided by the authorities, they blindly believe the false news compiled by some "We Media" and even spread it many times, causing public panic, which has brought obstacles to the effective prevention and control of the epidemic. This paper analyzes the main communication modes of network public opinion. There are many ways to spread public opinion information at present. New media such as TikTok and Facebook have changed the way people receive COVID-19 information, and the rapid development of $5 \mathrm{G}$ technology has provided an even more convenient channel for information dissemination.

The trend and characteristics of public opinion under the background of COVID-19 are mainly manifested as:

First, the cycle of public opinion is obviously long. The public opinion about COVID19 will also exist for a long time and become the focus of the public opinion field. In view of the normalization of COVID-19, if the public can't maintain a correct and positive attitude but listen to biased public opinion information, it will be a great challenge for the prevention and control of t COVID-19.

Second, there are many ways to spread public opinion information. The new media such as Tiktok and facebook have changed the way people receive information about COVID-19 [8]. Especially in the context of the current Internet, the rapid development of 5G technology provides a more convenient channel for information dissemination. Information in short video spreads fast, has wide audience and wide range, in a short period of time can have dozens or even millions of broadcast volume, can convey the real-time information related to COVID-19 to citizens of different ages in different social occupations. Because the information contains pictures, video and audio, compared with ordinary text information, it is more credible and easier to attract the attention of citizens. Once COVID19 information passes through the rapid spread of social media, it will definitely have a huge impact. Third, the centre of the public opinion field is focused, and the subject of the event is extremely active. From the rebound of COVID-19 in a certain place to the official announcement of the management and control department of a certain place that "the current situation of COVID-19 in the capital tends to be stable and better", netizens have a series of "soul torture" against COVID-19 in a certain place, including "why is the outbreak and the rebound of COVID-19 in a certain place?", and "Why is the rebound in the seafood market? Is there a serious problem in the circulation system of urban agricultural and sideline products?" COVID-19 in a certain place has been pushed to the climax and always stood at the centre of many public opinion events. However, compared with many obvious problems such as untimely disclosure of epidemic information, lack of medical resources, and poor nucleic acid detection ability, a certain place should respond quickly after the rebound of the epidemic, such as daily notification, immediate isolation, one-day locking of a certain place, two-day big data tracking and upgrading of regional risk level, three-day stable material supply, one-week rescheduling of emergency response, two-week emergency response Inner speed to enhance nucleic acid detection ability, always in the active position. It can be regarded as a model of "rapid response", "strong response" and "thunder prevention and control". With the early warning of COVID-19 prevention and control, a certain region has a clear understanding of the deployment of response measures at all levels. In addition, a large range of the best medical resources are gathered in this region and the effectiveness of various prevention and control measures is timely, objective and transparent in front of the public. This makes the positive speech steadily occupy the mainstream of public opinion on the Internet. 


\section{New business trend in the epidemic}

COVID-19 is spreading around the world, promoting digital upgrading, including online government, business, science, education, culture and health, and other organizations, operations and maintenance. The confinement measures and social isolation measures implemented to resist infectious diseases have proved the effectiveness of online office and life, and promoted the transformation of existing industrialization. E-commerce, medical robot, telecommuting or real-time health monitoring technology will be further developed in the future. Management and control departments need to promote the construction of digital government and smart city according to the characteristics of the times, and strengthen the Internet big data, artificial intelligence, digital government and other scientific and technological means in the epidemic prevention and control. In this epidemic prevention and control, Internet big data, artificial intelligence, digital government and other scientific and technological means have played a significant role, providing important support and guarantee for epidemic prevention and control. For example, some regions use big data such as telecommunications to strengthen the traceability and monitoring of epidemic situation, provide information on personnel flow, and support the research and judgment of epidemic situation and the deployment of epidemic prevention and control. However, the epidemic also exposed some problems, such as the lack of digital governance ability of individual local management and control departments, and the imbalance of regional application level of science and technology prevention and control. For example, in the process of epidemic prevention and control, information collection and sharing, material allocation, cross domain coordination, and government services in individual regions are mainly based on legs, mouths, and pens, thus reducing the efficiency of prevention and control.

To promote the construction of digital management and control department and smart city, it is necessary to break through the data barrier and make the urban data enabling social governance precise and refined. Research finds that in the new crown pneumonia epidemic prevention and control, the coordination and efficiency of the health sector with the public security, transportation, communications, medical supplies management departments, and surrounding cities and even communities have been challenged, which has further highlighted the cross departmental, The demand and urgency of data fusion between cities.

E-commerce has a solid foundation for development. After the outbreak of COVID-19, the way of home segregation increased the demand of residents for e-commerce. Many enterprises that already have online stores have hot sales business, while enterprises that only focus on physical stores have to create online stores urgently in the face of dismal offline sales. While the epidemic breeds a large number of online office business for enterprises, it also spawns new business formats, bringing about resource integration and business restructuring. For example, the first mock exam is being carried out by the epidemic situation and the resumption of production and recovery. Enterprises are actively exploring the "shared employee" mode, and many local control departments are actively promoting this mode. Some districts promoted the "shared employees" model, relying on industry associations to help enterprises build a communication platform and promote more temporarily unemployed employees to work as soon as possible. It can be predicted that with the increase of sharing enterprises, there may be platform enterprises specializing in the management of "sharing employees", and "sharing employees" will develop into a more flexible employment mechanism.

Online education, online entertainment, online video and other public oriented industries also developed rapidly during the period of epidemic prevention and control. For example, many Internet online education have rapidly attracted many users by providing 
live broadcast services or building interactive platforms for public schools and offline educational institutions.

\section{Conclusion}

The digitalization of enterprises has become a focus of public opinion. After the outbreak of COVID-19, the operation of the social line was blocked, and the application of online workers and teleconferencing was developing rapidly. The importance of telecommuting has long been regarded as an alternative production method to improve the enterprise process, but the vast majority of enterprises do not have telecommuting projects.

In order to cope with the impact, the industry has actively explored the use of industrial Internet technology to help enterprises accurately connect with customers, realize online collaborative operation, improve enterprise management ability and decision-making ability, and accelerate the recovery of the manufacturing industry, according to the people's post and Telecommunications daily. Digitalization is the key anti vulnerability capability of enterprises, and online and digitalization are the inevitable trend of all walks of life.

This work was supported by the National Social Science Foundation of China (18ZDA309), the National Natural Science Foundation of China (62072463, 71531012), the Opening Project of State Key Laboratory of Digital Publishing Technology of Founder Group (413217003).

\section{References}

1. There have been nine financial crises in history, and the whole world has been affected. $\begin{array}{llll}\text { Retrieved July } & 29, & \text { from }\end{array}$ http://www.360doc.com/content/19/0729/22/33209086_851764484.shtml

2. The economic crisis. Retrieved from https://baike.baidu.com/item/\%E7\%BB\%8F\%E6\%B5\%8E\%E5\%8D\%B1\%E6\%9C\% BA/631674? fr=aladdin

3. Yuqi Zhang, Huan Wang, KexinZeng. Impact of COVID-19 on China's regional economy. Journal of Hubei University of Economics (Humanities and Social Sciences),2021,18(04):47-49.

4. Youxin Wang, Yifan Wang, Hanfang Yang. Types of External Shock and China's Economic Cycle Fluctuation -- Also on the Effectiveness of Macroprudential Policies. International financial research,2021(03):14-26.

5. Lilin Zhao. Influences of COVID-19 on China's economy and industries. Trade show economic, 2021(03):41-43.

6. Qinjun Lu, Shuoyue Fen, Ting Su. During the fight against COVID-19, the "Internet Plus Medical Service" has demonstrated its advantages. Health care for middle aged and elderly,2020(12):8-9.

7. Yin Chen. Thinking on the educational mode caused by public emergencies. Journal of higher education,2021(10):62-65.

8. ZhongHua Ning. Social media and public opinion response in COVID-19 epidemic [J]. Research on Information Security,2021,7(02):155-165. 